\title{
Globe
}

Revue internationale d'études québécoises

\section{Michel Plourde [éd.], avec la collaboration de Hélène Duval et de Pierre Georgeault : Le français au Québec. 400 ans d'histoire et de vie. [Montréal], Fides-Publications du Québec, 2000}

\section{Rainier Grutman}

Volume 6, numéro 2, 2003

URI : https://id.erudit.org/iderudit/1000823ar

DOI : https://doi.org/10.7202/1000823ar

Aller au sommaire du numéro

Éditeur(s)

Globe, Revue internationale d'études québécoises

ISSN

1481-5869 (imprimé)

1923-8231 (numérique)

Découvrir la revue

Citer ce compte rendu

Grutman, R. (2003). Compte rendu de [Michel Plourde [éd.], avec la collaboration de Hélène Duval et de Pierre Georgeault : Le français au Québec. 400 ans d'histoire et de vie. [Montréal], Fides-Publications du Québec, 2000].

Globe, 6(2), 179-181. https://doi.org/10.7202/1000823ar d'utilisation que vous pouvez consulter en ligne.

https://apropos.erudit.org/fr/usagers/politique-dutilisation/ 
Quatre périodes historiques, quatre formes de dialogues culturels... On pourrait certes reprocher aux auteurs la rigidité de leur méthode et de leurs catégories. Ne négligent-ils pas les contradictions et les nuances des textes, œuvres singulières que l'on ne saurait ranger d'emblée sous une bannière générique? Si les analyses semblent parfois rapidement esquissées, fondées le plus souvent sur l'élaboration de réseaux thématiques, cela tient à la perspective adoptée, celle de l'histoire littéraire. Car, comme toute histoire, celle de Moisan et de Hildebrand organise la matière livresque, présente une périodisation et des concepts généraux, rigides sans doute, mais voués tout de même à la composition d'une vue panoramique. Question de rendre justice au travail minutieux des auteurs, précisons que l'histoire qui nous est racontée est de nature savante : elle repose sur une recherche rigoureuse et méthodique; elle prend en compte les études les plus récentes sur l'identitaire et s'appuie notamment sur la Banque de données d'histoire littéraire québécoise et néo-québécoise, outil informatique permettant de colliger les différents thèmes explorés par les écrivains migrants. Mené avec rigueur, Ces étrangers du dedans. Une histoire de l'écriture migrante au Québec (19371997) constituera sans doute un outil de référence pour les chercheurs intéressés par le sujet. L'ouvrage, cependant, possède les lacunes de l'étude pionnière : oscillant entre le général et le particulier, il embrasse une vaste problématique sans toujours réussir à en traduire la richesse et la diversité.

Martine-Emmanuelle Lapointe

Université de Montréal

\section{Michel Plourde [éd.], avec la collaboration de Hélène Duval et de Pierre Georgeault \\ Le français au Québec. 400 ans d'bistoire et de vie. [Montréal], Fides-Publications du Québec, 2000.}

Magnifiquement illustré, assorti d'une bibliographie substantielle, de deux index et d'une chronologie fort détaillée, ce volume s'adresse au public averti comme à l'amateur éclairé, désireux de voir une communauté de langue française décliner les multiples facettes de son identité. La matière a été répartie sur quatre volets chronologiques. Le premier, qui est aussi le plus court (p. 1-52), évoque le « statut royal " du français 
à l'époque de la Nouvelle-France (1608-1760); le deuxième (p. 55-132) raconte les combats d' "une langue sans statut " en Amérique du Nord britannique, mais "qui ne capitule pas. (1760-1850); le troisième (p. 135-229) décrit le "statut compromis " du français au sein de la Confédération canadienne, malgré (ou à cause de?) l'idéologie de conservation qui domine les années 1850-1960. Le quatrième et dernier volet, le plus important du point de vue quantitatif (p. 232-429, soit près de la moitié du volume) célèbre le renversement du transfert linguistique vers l'anglais et " la reconquête du français " (1960-2000).

La formule du collectif était tout indiquée pour une pareille somme de travail - l'époque est en effet révolue où Ferdinand Brunot pouvait prétendre rédiger seul sa monumentale Histoire de la langue française (1916-1938). Ce qui frappe d'emblée le lecteur de cet ouvrage dirigé par Michel Plourde, ancien président du Conseil de la langue française, c'est qu'il ne s'agit pas uniquement d'un travail de linguistes. Le comité scientifique a visé l'interdisciplinarité. Parmi les disciplines mises à contribution, il y a tout d'abord l'histoire (Micheline Dumont, René Durocher, Claude Galarneau, Marc V. Levine, Jean-Claude Robert, Nive Voisine), ensuite la sociologie (Denys Delâge, Simon Langlois, Guy Rocher, Joseph Yvon Thériault) et les sciences politiques (Alain G. Gagnon), enfin, dans une moindre mesure, la démographie et la géographie. La présence d'écrivains (Marcel Dubé, Naïm Kattan, Monique LaRue, Marco Micone, Émile Ollivier) et de critiques littéraires s'explique par l'investissement symbolique considérable dont la langue française a fait et continue de faire l'objet dans la littérature québécoise : Maurice Lemire et Marie-Andrée Beaudet le montrent pour les époques plus reculées, André Brochu et Lise Gauvin pour l'euphorie de la Révolution tranquille et la dépression post-référendaire (1980).

Une palette de collaborateurs aussii riche permet sans conteste de multiplier les points de vue : un juriste (José Woehrling) complétera ainsi les pages passionnées d'un sociologue (Guy Rocher) sur la Charte de la langue française, dite Loi 101. Ce jalon de l'histoire politique du Québec me fournira pourtant l'occasion de formuler une réserve. Loin de moi l'idée de contester l'importance de la loi en question : si le Québec actuel est ce qu'il est, c'est en bonne partie grâce à l'application des principes qui y sont formulés. Qu'on lui consacre un chapitre (p. 273-299) me paraît donc justifié. Ma réserve concerne davantage la répartition des sujets de l'ouvrage en généra! : il me semble que les faits qui relèvent de 
ce qu'on appelait traditionnellement * l'histoire externe - d'une langue (le contexte socio-économique, le cadre politique), faits qui influent sans aucun doute sur son statut, laissent dans l'ombre les éléments qui pourraient servir à une "histoire interne " (son évolution sur les plans phonétique, morphologique, syntaxique). Non pas que ces derniers éléments soient tout à fait absents de l'ouvrage que voici; mais ils ne m'y paraissent pas recevoir toute l'attention qu'ils mériteraient dans une histoire linguistique (même si elle ne prétend pas être une histoire de la langue en tant que telle). On regrettera d'autant plus ce déséquilibre que c'est notamment par les traits formels de son corpus que le français du Québec se distingue de celui de tant d'autres régions de la francophonie.

Cette distinction, on le sait, est avant tout d'ordre phonétique et lexical. Les Québécois ont gardé des traces de l'accent normand de leurs ancêtres (Tocqueville l'avait noté dès 1831, dans une observation lucide, faite parmi plusieurs remarques désobligeantes). Certains de ces traits se sont maintenus et généralisés au point de fonder une norme orale locale, qu'on aurait tort de confondre avec l'usage socialement stigmatisé du joual. La description synchronique du " français standard d'ici " n'en est toutefois qu'à ses débuts : l'article de Pierre Martel et d'Hélène CajoletLaganière (p. 379-388) en donne un bon aperçu. Le matériel disponible pour reconstituer la diachronie de ladite norme (on songe aux correspondances privées, sur lesquelles travaille ma collègue France Martineau) n'a guère été exploité jusqu'à présent : on trouvera ici des mises au point utiles sur l'homogénéisation du français en Nouvelle-France (Raymond Mougeon, p. 33-38) et sur le français des premiers Canadiens (Jean-Denis Gendron, p. 39-42). Avec l'accent, c'est probablement le lexique qui distingue le plus le français québécois : cela explique les références fréquentes à des questions de vocabulaire, de toponymie, de terminologie (Marthe Faribault, p. 19-20 et 210-211, Claude Poirier, p. 111-119 et 222, Chantal Bouchard, p. 197-199, Louis Mercier, p. 206209). Si de telles contributions, faites par des chercheurs qui étudient la langue pour elle-même, avaient été plus nombreuses et surtout plus étoffées (mises ensemble, celles que j'ai signalées dépassent à peine la longueur du chapitre consacré au projet de la Loi 101), on aurait pu parler d'une histoire du français $d u$ Québec, ouvrage qui fait malheureusement encore défaut après la parution de cette belle et instructive histoire du Français au Québec.

Rainier Grutman Université d'Ottawa 